\title{
Need for Digital Biomarkers in Musculoskeletal Trials
}

\author{
Jörg Goldhahn \\ Institute for Translational Medicine, ETH Zurich, Zurich, Switzerland
}

\section{Keywords}

Musculoskeletal trial · Digital biomarker

\section{Abstract}

Pain and loss of function - both problems cause patients to visit a musculoskeletal specialist. Therefore, both lead symptoms should serve as a benchmark for new therapeutic approaches. New technologies generating digital biomarkers have the potential to significantly change musculoskeletal trials. However, more work is needed to agree upon data and variable standards, to improve user friendliness, and to ensure data integrity along the whole processing way. Therefore, rigorous and systematic testing of new technological approaches is required to establish new outcome variables suitable for musculoskeletal trials. Consortia of researchers working on similar technologies and outcome variables should collaborate from the beginning to enable comparing and pooling data. Early interaction with health authorities and regulatory bodies are necessary to pave the way for a widespread use of a new technology.

\section{The Need}

Pain and loss of function - both problems cause patients to visit a musculoskeletal specialist. Therefore, both lead symptoms should serve as a benchmark for new therapeutic approaches. In other words, can we eradicate the pain and re-establish full function of the movement system? While pain is still a very subjective measure, musculoskeletal function can be assessed and quantified with two different measurement tools: asking the patients about certain functions or let patients perform them.

The first option results in patient-reported outcome (PRO). Systematic research in the last few years has broadened our understanding about the potential and limitations of this 


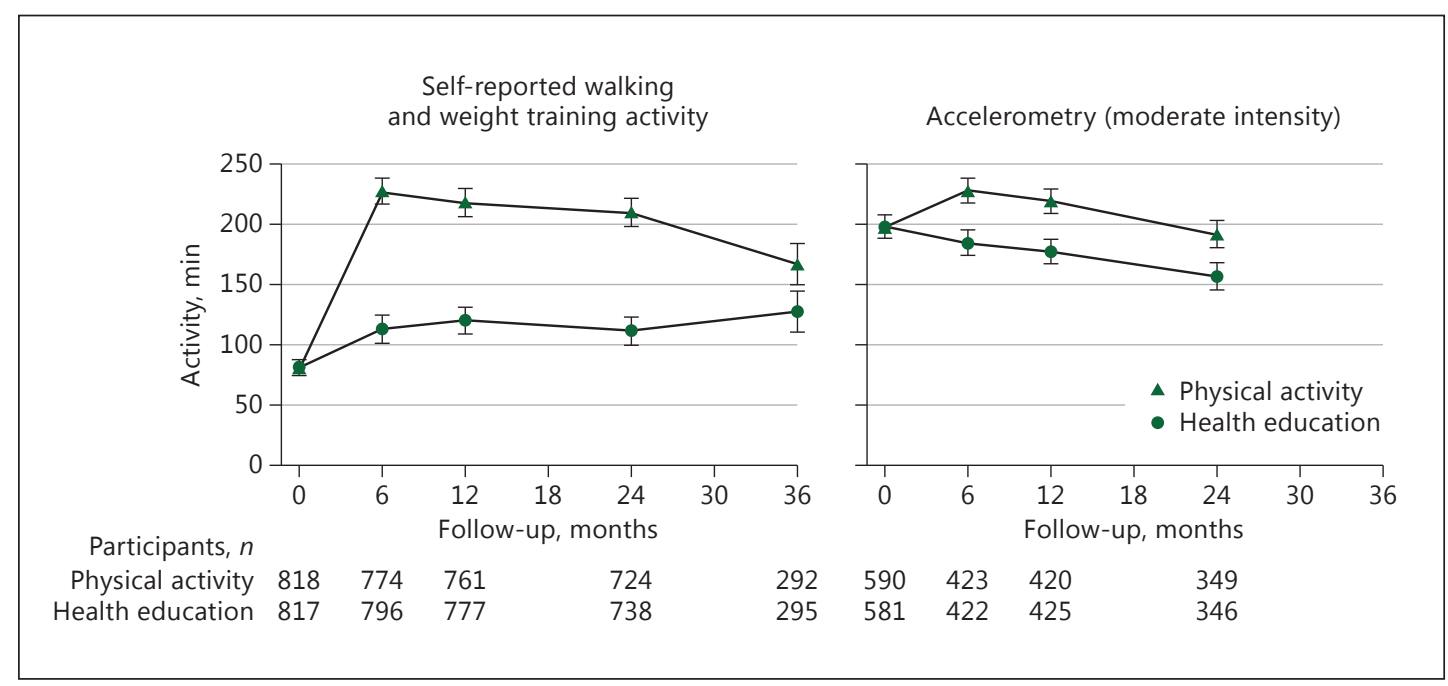

Fig. 1. Difference between self-reported walking and weight training activities and real-world moderateintensity activity measured with accelerometry in the LIFE study by Pahor et al. [6]; reproduction with permission of Pahor et al.

measurement tool. PRO using questionnaires is considered a subjective measure [1]. The clear advantage is that patients describe their functional status in relation to activities of daily living and quality of life. Therefore, the resulting scores are highly relevant to patients (high face validity). Psychometric evaluations reveal that many scores can be used to measure changes in disease status (high sensitivity to change) with high reproducibility in patients (high intraindividual reliability). However, they can be heavily confounded by patient perception and vary significantly between patients (high interindividual variability) [2]. For example, 5,000 steps per day can result in a very high subjective score of an elderly lady shortly after a hip fracture. In contrast, it would lead to a very unsatisfactory score in a young, otherwise healthy patient because it may represent only $50 \%$ of his/her usual performance.

Functional tests represent another option to quantify musculoskeletal function. These tests are considered objective measures [3]. Patients are asked to perform typical movements or tasks, which are measured or rated by the examiner. Advantages of performance tests include quantitative description of function, objective external assessment, and high sensitivity to change [4]. However, performance tests can be heavily confounded by the environment including the examiner as well as equipment but also by day-to-day variability. Their relevance for patients is often questionable because they are performed under lab conditions. A good illustration is the gait speed test. It is an inexpensive test, where patients are instructed to "walk at a comfortable pace" over a short distance and the mean speed is calculated using $5 \mathrm{~m}$ as a base. Although the test is considered a core indicator of health and functioning in disease and ageing, a number of confounding variables like test environment, instructor differences, or day-to-day variability limit the applicability of the test [5].

\section{Musculoskeletal Digital Biomarker}

New technological solutions such as accelerometers and other motion sensors enable quantification of many different types of movement and mobility - not only under lab conditions but also in real life. Examples range from simple gait counters to complex technologies 


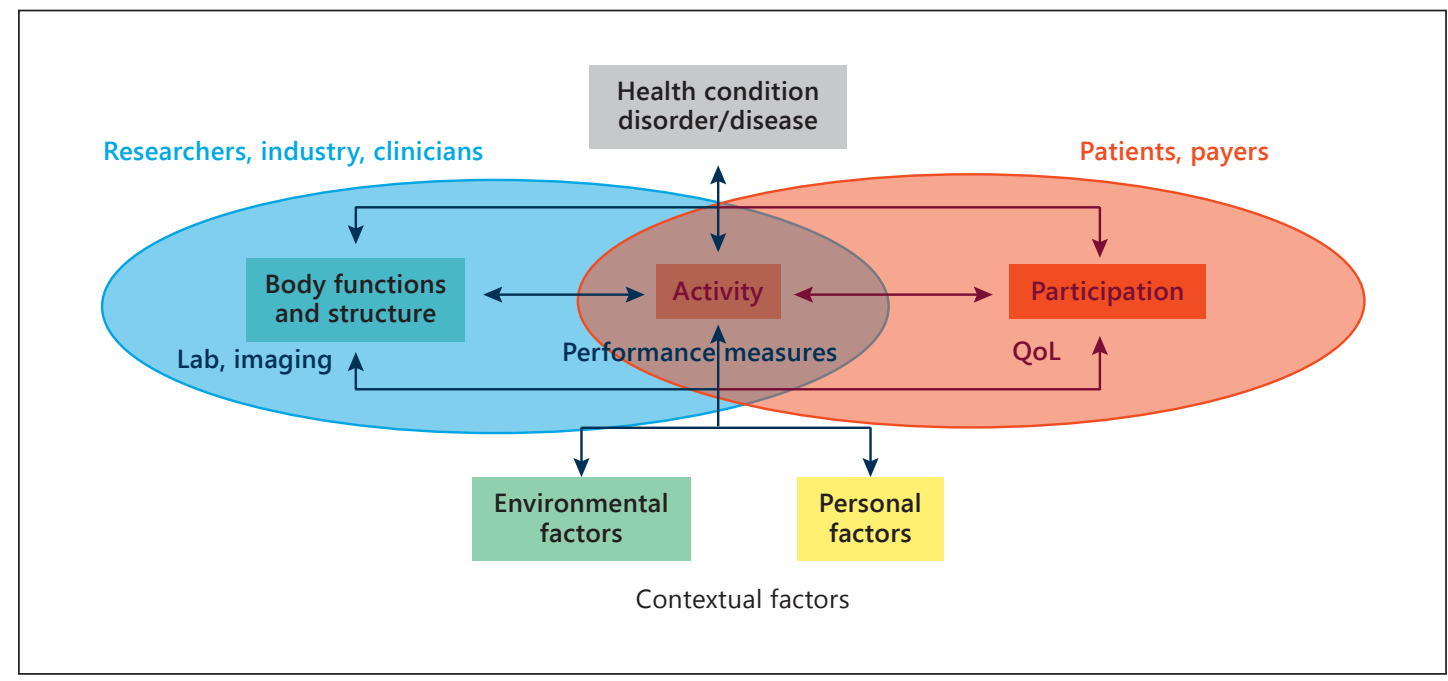

Fig. 2. Interest of different stakeholders in the WHO International Classification of Functioning, Disability and Health (ICF), modified after: Towards a Common Language for Functioning, Disability and Health. ICF. Geneva, WHO, 2002. QoL, quality of life.

that can detect falls or upper extremity movement. These technologies can quantify real-life function in contrast to perceived function as measured with questionnaires (higher face validity). While PROs are confounded by patient expectation, technologies that measure physical performance in real life are not. An impressive illustration of this finding has been observed in the LIFE study by Pahor et al. [6]. They tested the hypothesis that a long-term structured physical activity program is more effective than a health education program in reducing the risk of major mobility disability using self-reported walking and weight training activities and accelerometry-derived moderate intensity. While self-reported activities revealed a positive effect for both interventions, accelerometry-based data revealed another scenario. They indicate a short transient effect of the physical activity program and a decline for the health education intervention (Fig. 1). This study illustrates that new technologies such as accelerometry are less confounded than PROs, and demonstrate higher face validity than PROs and have the potential to better characterize all domains of the ICF (International Classification of Functioning, Disability and Health) disease models than traditional instruments (Fig. 2). In addition, wearable devices can be utilized for remote monitoring, thereby reducing the number of necessary follow-up visits while generating more data points.

\section{Digital Biomarkers in Musculoskeletal Trials}

New technologies generating digital biomarkers have the potential to change musculoskeletal trials significantly. They generate new information closer to real life, such as real-life gait speed, falls within the individual environment, and upper extremity movement patterns during the day. Mobile monitoring using wearable devices enable continuous data streaming. The resulting data provide a wealth of additional data points, may decrease the need for follow-up visits, and may serve as a base for complex data processing such as movement pattern analyses. Successful applications include quantification of significant changes in gait quantity and quality under a constrained environment [7], recovery of shoulder function after rotator cuff repair [8] and prediction of falls with accelerometry-based balance quanti- 
Goldhahn: Need for Digital Biomarkers in Musculoskeletal Trials

fication [9]. The combination with either data such as vital signs but also positioning data adds meaning to the pure biomechanical data. For example, the combination of positioning data (derived from GPS) with accelerometry data and vital parameters may explain gait adaptation and stumbling during uphill working resulting in shortness of breath.

Several pilot trials have demonstrated that new technology may generate digital biomarkers for musculoskeletal trials. However, more work is needed to agree upon data and variable standards, to improve user friendliness, and to ensure data integrity along the whole processing way. Therefore, rigorous and systematic testing of new technological approaches is required to establish new outcome variables suitable for musculoskeletal trials. They have to be established using standard guidelines for new diagnostic devices and outcome variables [10]. This includes characterization of face validity, accuracy, and reproducibility. On top, the complete data processing has to be described. This includes data generation, storage, processing, uploading into central systems, and calculation of aggregate variables [11]. It is crucial to characterize patient compliance with the technology, for example, with wear time. Data, such as total amount of steps per day, are not relevant without this additional information [12]. Data privacy has to be ensured during the complete process. Special care is necessary to document hardware versions as well as software updates. Comparability of data across different hardware and software versions needs to be documented. A compelling case is required to convince health authorities and payers to make use of the new technology. Therefore, studies are needed demonstrating that new variables are used for therapeutic decision-making that the number follow-up visits in a study can be decreased or additional data generated lead to new treatment approaches.

Consortia of researchers working on similar technologies and outcome variables should collaborate from the beginning to enable comparing and pooling of data [13]. Initiatives like COMET (Core Outcome Measures in Effectiveness Trials) can help define standards in clinical trials. Finally, early interaction with health authorities and regulatory bodies are necessary to pave the way for a widespread use of a new technology.

\section{Conflict of Interest Statement}

The author declares no conflict of interest

\section{References}

$>1$ Basch E, Abernethy AP, Reeve BB: Assuring the patient centeredness of patient-reported outcomes: content validity in medical product development and comparative effectiveness research. Value Health 2011;14:965966.

-2 Macefield RC, Jacobs M, Korfage IJ, Nicklin J, Whistance RN, Brookes ST, Sprangers MA, Blazeby JM: Developing core outcomes sets: methods for identifying and including patient-reported outcomes (PROs). Trials 2014; $15: 49$.

3 Nightingale EJ, Pourkazemi F, Hiller CE: Systematic review of timed stair tests. J Rehabil Res Dev 2014;51:335350.

-4 Bisca GW, Morita AA, Hernandes NA, Probst VS, Pitta F: Simple lower limb functional tests in patients with chronic obstructive pulmonary disease: a systematic review. Arch Phys Med Rehabil 2015;96:2221-2230.

5 Peel NM, Kuys SS, Klein K: Gait speed as a measure in geriatric assessment in clinical settings: a systematic review. J Gerontol A Biol Sci Med Sci 2013;68:39-46.

6 Pahor M, Guralnik JM, Ambrosius WT, Blair S, Bonds DE, Church TS, Espeland MA, Fielding RA, Gill TM, Groessl EJ, King AC, Kritchevsky SB, Manini TM, McDermott MM, Miller ME, Newman AB, Rejeski WJ, Sink KM, Williamson JD; LIFE study investigators: Effect of structured physical activity on prevention of major mobility disability in older adults: the LIFE study randomized clinical trial. JAMA 2014;311:2387-2396. 
7 Belavý DL, Gast U, Daumer M, Fomina E, Rawer R, Schiessl H, Schneider S, Schubert H, Soaz C, Felsenberg D: Progressive adaptation in physical activity and neuromuscular performance during 520d confinement. PLoS One 2013;8:e60090.

$\checkmark 8$ Pichonnaz C, Duc C, Jolles BM, Aminian K, Bassin JP, Farron A: Alteration and recovery of arm usage in daily activities after rotator cuff surgery. J Shoulder Elbow Surg 2015;24:1346-1352.

-9 O'Sullivan M, Blake C, Cunningham C, Boyle G, Finucane C: Correlation of accelerometry with clinical balance tests in older fallers and non-fallers. Age Ageing 2009;38:308-313.

10 Williamson PR, Altman DG, Blazeby JM, Clarke M, Devane D, Gargon E, Tugwell P: Developing core outcome sets for clinical trials: issues to consider. Trials 2012;13:132.

11 Lee IM, Shiroma EJ: Using accelerometers to measure physical activity in large-scale epidemiological studies: issues and challenges. Br J Sports Med 2014;48:197-201.

12 Keadle SK, Shiroma EJ, Freedson PS, Lee IM: Impact of accelerometer data processing decisions on the sample size, wear time and physical activity level of a large cohort study. BMC Public Health 2014;14:1210.

13 Williamson P, Altman D, Blazeby J, Clarke M, Gargon E: Driving up the quality and relevance of research through the use of agreed core outcomes. J Health Serv Res Policy 2012;17:1-2. 